\title{
Ogilvie Syndrome Following Caesarean Section: A Case Report
}

\author{
Sanae Sninate ${ }^{1,2^{*}}$, Habib Bellamlih ${ }^{1,2}$, Soukaina Allioui ${ }^{1,2}$, Leila Jroundi ${ }^{1,2}$, \\ Fatima Zahrae Laamrani ${ }^{1,2}$
}

\begin{abstract} essential to avoid cecal perforation. without transitional mechanical obstruction. The patient was successfully treated. who present with pain, severe abdominal distension and failure to pass flatus after caesarean section.

\section{Keywords}

Ogilvie Syndrome; Caesarean Section; Acute Colonic Pseudo-Obstruction; CT Scan

${ }^{1}$ Emergency Radiology Department, Ibn Sina Hospital, Mohammed V University, Rabat, Morocco

${ }^{2}$ Medicine and Pharmacy of Rabat, Mohammed V University, Rabat, Morocco

*Corresponding author: sninate.sanae@gmail.com
\end{abstract}

Background. Ogilvie syndrome is a rare postpartum complication. It is characterized by an acute colonic pseudo-obstruction which occurs in the absence of a mechanical cause. Early detection of the diagnosis is

Case report. We report a case of Ogilvie syndrome following caesarean section in a 39-year-old woman (gravida 2, para 2) with a history of secondary infertility 10 years ago due to tubal stenosis and subserous fibroma, which led to the indication for cesarean section in second pregnancy at 39 weeks of gestation. Three days after cesarean section, the patient presented with significant abdominal distension and tenderness, vomiting, weakness, and nausea; an abdominal X-ray showed cecal distension. Abdominal computed tomography scan with intravenous contrast revealed distension of the ascending and transverse colon with air-fluid levels but

Conclusions. Ogilvie syndrome is a rare but serious complication that should be considered in fit young patients

\section{Background}

Ogilvie syndrome, or acute colonic pseudo-obstruction (ACPO), was first described in 1948 by Sir William Ogilvie. It is characterised by a functional colonic obstruction for reasons that remain poorly understood [1]. This is an acute massive dilation of the colon without mechanical obstruction. The condition must be treated appropriately to avoid cecal perforation. We report a case of Ogilvie syndrome following caesarean section in a 39-year-old female patient who was successfully treated.

\section{Case Report}

The patient was a 39-year-old woman (gravida 2, para 2) with a history of secondary infertility 10 years ago due to tubal stenosis and subserous fibroma which led to the indication for cesarean section in second pregnancy at 39 weeks of gestation. Three days after cesarean section, the patient presented to the emergency department with significant abdominal distension and tenderness, vomiting, weakness, and nausea. Physical examination revealed abdominal distension, absent bowel sounds, superficial and deep pain during palpation, negative sign of peritoneal irritation, generalized bloating consistent with bowel obstruction. Laboratory findings revealed hypokalemia and elevation of C-reactive protein (CRP), the remaining laboratory results were unremarkable.

Abdominal X-ray (Fig. 1) showed cecal distension without pneumoperitoneum, calcifications, and air-fluid levels. Abdominal computed tomography (CT) with intravenous contrast revealed distension of both the ascending and transverse colon with air-fluid levels (Fig. 2). The descending and sigmoid colon showed no sign of distension. No transitional mechanical obstacle could be found. Furthermore, the presence of pneumoperitoneum was to be seen due to the history of her recent caesarean section. The diameter of the caecum measured $9 \mathrm{~cm}$. 


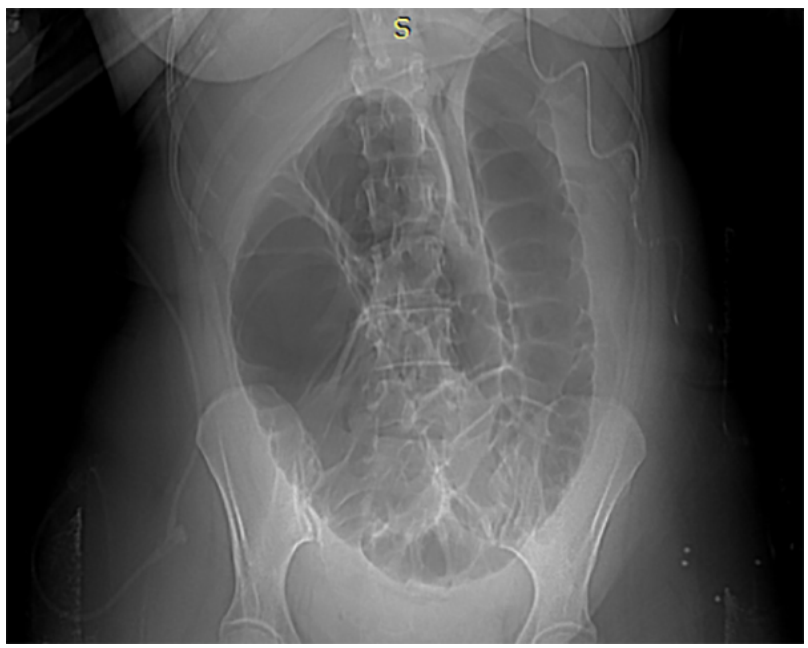

Figure 1. Cecal distension without pneumoperitoneum, calcifications and air-fluid levels.

The patient was treated with electrolyte administration, nasogastric decompression, Foley and central venous catheters in combination with pharmacological treatment including intravenous bolus administration of neostigmine.

The woman received two weeks of inpatient observation with a gradual improvement and normalization of laboratory results.

\section{Discussion}

Ogilvie syndrome is a rare postsurgical complication, which can be associated with cesarean section. Clinically, it presents with abdominal distension and massive dilatation of the colon, without mechanical obstruction [2].

The evolution may be towards stabilization or reduction in gas distension spontaneously or after treatment, but there is a risk of recurrence despite several days of favorable clinical course.

The major risk is cecal perforation complicated by stercoral peritonitis even after several days of favorable clinical course. According to the authors [3], its frequency varies between 25 and $40 \%$. The pathophysiology is not fully understood; however, an imbalance in autonomic innervation of the colon is believed to be the cause [4]. The incontinent ileocecal valve can protect the cecum from overdistension allowing the gases to pass through the small intestine with cecal perforation occurring only on the Bauhin's valve.

After cesarean section, there are several important symptoms and signs that can indicate the presence of Ogilvie syndrome. However, at the early stage, these symptoms and signs may be similar to those indicating normal postoperative course and ileus, while signs occurring later may mimic mechanical bowel obstruction, or intestinal damage and leakage of bowel content secondary to the initial operation [5]. The average duration of the appearance of the first symptoms in Ogilvie syndrome is 48 hours, but it may be as early as 6 hours after

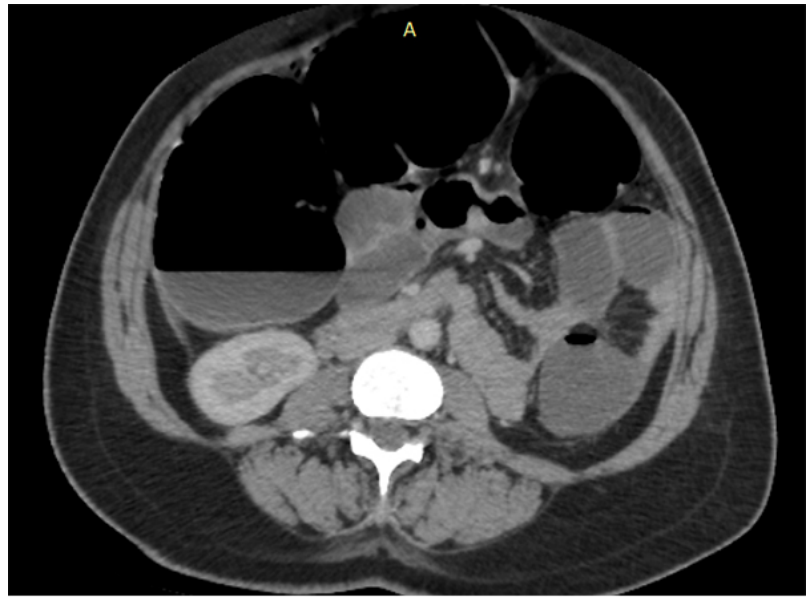

(A)

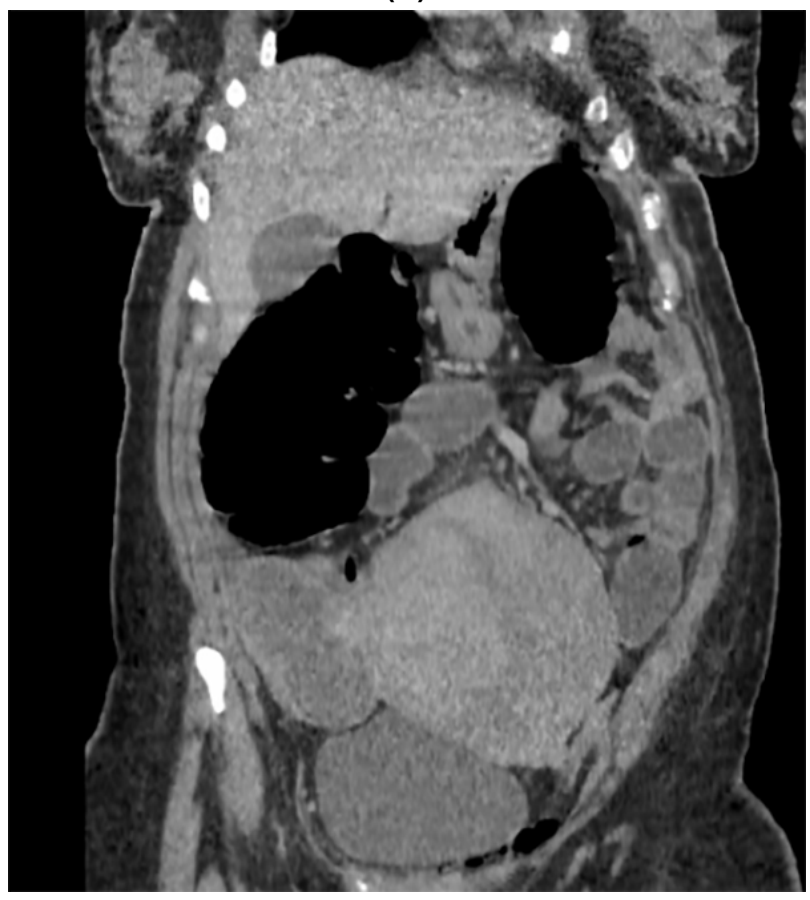

(B)

Figure 2. Axial (A) et coronal (B) CT scan abdominal with intravenouse contrast revealed distension of the ascending and transverse colon with air-fluid levels without transitional mechanical obstacle.

delivery [6]. There are some clinical features that may support the diagnosis of Ogilvie syndrome rather than other diagnoses. They include significant abdominal distension with minimal nasogastric aspirations, some degree of bowel movement (diarrhea or the presence of bowel sounds), low fever or increase in CRP with no obvious source of infection [5]. There is a difficulty to differentiate ileus and Ogilvie syndrome, but ileus is characterized by a lesser degree of abdominal distension and is more likely to be associated with no bowel sounds and no passage of flatus [5]. 
The pathogenic factors include prolonged labor, maternal fatigue and weight loss, poor general health status, fluid loss, hypoalbuminemia, postoperative analgesia, and electrolyte imbalance or acid-base balance disorder [7].

The firstline of imaging is abdominal X-ray. It may show an overall colonic distension extending from the cecum to the rectosigmoid junction or part of the colon. However, it always predominates at the level of the cecum.

The preservation of colonic haustrations, the rarity of hydro-air levels and the presence of gas within the rectum tend to suggest a functional occlusion, which should result in the diagnosis of Ogilvie syndrome.

On the other hand, the X-ray of the abdomen is of little help in diagnosing cecal perforation as the pneumoperitoneum is inconstant, so that its presence is physiological up to five days after laparotomy.

Contrast-enhanced abdominal CT should be performed to eliminate some differential diagnoses such as mechanical obstruction, sigmoid or cecum volvulus, fecal impaction or peritonitis. Colectasia usually begins in the cecum and the right colon and extends downstream to a point of incongruence called the cut-off [8].

Cecal diameter can be more accurately measured with CT and can be useful in guiding management: the diameter of the cecum greater than $14 \mathrm{~cm}$ is associated with a greater risk of perforation and mortality [5]. However, cecal diameter alone should not determine the management as there is variation in the risk of perforation according to cecal diameter in cases of perforation below $9 \mathrm{~cm}$ in women after caesarean section [9].

Therapy is based on several treatment modalities. It is assumed that when the cecum diameter is less than $9 \mathrm{~cm}$, patients can undergo treatment with a sympathetic blocker (guanethidine) and acetylcholinesterase inhibitor (neostigmine) first. Colonoscopic exsufflation often helps obtain the decompression of the colon, but carries significant risks for perforation. In case of perforation or intestinal distress, surgery is obviously the standard treatment.

\section{Conclusions}

Ogilvie syndrome is a rare condition that results in acute colonic pseudo-obstruction in the postpartum period. It should be considered in fit young patients who develop pain, severe abdominal distension and failure to pass flatus after caesarean section. The early detection of the diagnosis allows to avoid complications and requires a multidisciplinary approach for better outcome.

\section{Ethical Statement}

The study was conducted according to WHO Declaration of Helsinki - Ethical Principles for Medical Research Involving Human Subjects.

\section{Informed Consent}

Written informed consent was obtained from the patient who participated in this study.

\section{Conflict of Interest}

The authors declare that no conflicts exist.

\section{Financial Disclosure}

The authors declared no financial support.

\section{References}

[1] Karaman K, Tanoglu A, Beyazit Y, Han I. Giant colonic volvulus due to colonic pseudo-obstruction. Case Reports [Internet]. 2015;2015(feb25 1):bcr2014207963. Available from: https://doi.org/10.1136/bcr-2014-207963

[2] Kotsev S. Ogilvie's syndrome following cesarean delivery: the Dubai's case. Saudi Journal of Anaesthesia [Internet]. 2011;5(3):335. Available from: https://doi.org/10.4103/1658-354X.84117

[3] Mainguy Le Gallou C, Eboué C, Vardon D, Von Théobald P, Dreyfus M. Ogilvie's syndrome following cesarean section: just think! Report of two cases and review of the literature. Journal de Gynécologie Obstétrique et Biologie de la Reproduction [Internet]. 2011;40(6):557-563. Available from: https://doi.org/10.1016/j.jgyn.2010.11.010

[4] Platt JA, Rossi CM. Ogilvie syndrome after cesarean section. Journal of the American Academy of Physician Assistants [Internet]. 2015;28(12):1. Available from: https://doi.org/10.1097/01.JAA.0000471302.71597.95

[5] Hughes AE, Smart NJ, Daniels IR. Acute colonic pseudoobstruction after caesarean section: a review and recommended management algorithm. The Obstetrician \& Gynaecologist [Internet]. 2019;21(4):283-290. Available from: https://doi.org/10.1111/tog.12602

[6] Sunnoqrot N, Reilly RF. Hypokalemia associated with colonic pseudo-obstruction (Ogilvie's syndrome). Case Reports in Nephrology and Dialysis [Internet]. 2015;5(2):118-123. Available from: https://doi.org/10.1159/000431086

[7] Peng Y, Peng C. Acute colonic pseudo-obstruction with bowel rupture after caesarean section in HELLP syndrome: a case report. BMC Pregnancy and Childbirth [Internet]. 2020;20(1). Available from: https://doi.org/10.1186/s12884-020-03414-9

[8] Jain D, Moussa K, Tandon M, Culpepper-Morgan J, Proctor DD. Role of interstitial cells of cajal in motility disorders of the bowel. The American Journal of Gastroenterology [Internet]. 2003;98(3):618-624. Available from: https://doi.org/10.1111/j.1572-0241.2003.07295.x 
[9] Jayaram P, Mohan M, Lindow S, Konje J. Postpartum acute colonic pseudo-obstruction (Ogilvie's syndrome): a systematic review of case reports and case series. European Journal of Obstetrics \& Gynecology and Reproductive Biology [Internet]. 2017;214:145-149. Available from: https://doi.org/10.1016/j.ejogrb.2017.04.028
Received: $2021-02-10$

Revised: 2021-03-26

Accepted: 2021-04-07 